\title{
Épidémie de virus Zika en Amérique latine : quels enjeux pour la Guyane française en avril 2016 ?
}

\section{Zika virus outbreak in Latin America: what are the challenges for French Guiana in April 2016?}

\author{
L. Epelboin - M. Douine $\cdot$ G. Carles $\cdot$ N. Villemant $\cdot$ M. Nacher $\cdot$ D. Rousset $\cdot$ F. Djossou $\cdot$ E. Mosnier \\ Reçu le 22 février 2016; accepté le 18 mars 2016 \\ (C) Société de pathologie exotique et Lavoisier SAS 2016
}

Résumé Débutée en 2015 au Brésil, une épidémie liée à un arbovirus peu connu, le virus Zika (ZIKV), sévit dans toute l'Amérique latine. Ce virus, considéré jusqu'à récemment comme un virus bénin, a révélé des complications jusqu'alors insoupçonnées : manifestations neurologiques graves chez l'adulte et malformations du système nerveux central chez les nouveau-nés de mère atteintes pendant la grossesse. Alors que le continent est plutôt habitué aux épidémies d'arbovirus, les complications suspectées et les nombreuses inconnues de ce dernier arrivant posent de nombreuses questions de santé publique. La Guyane française, département français situé au nord-est du continent sud-américain, associe à la fois des ressources de niveau européen et un climat et des problématiques

L. Epelboin $(\bowtie) \cdot$ F. Djossou $\cdot$ E. Mosnier

Unité des maladies infectieuses et tropicales,

Centre hospitalier Andrée Rosemon, Cayenne, Guyane française

e-mail : epelboincrh@hotmail.fr

L. Epelboin $\cdot$ M. Douine $\cdot$ M. Nacher $\cdot$ F. Djossou $\cdot$ E. Mosnier Ecosystèmes amazoniens et pathologie tropicale (EPAT).EA3593. Université de la Guyane. Guyane Française

M. Douine $\cdot$ M. Nacher

Centre d'investigation clinique Antilles-Guyane,

Inserm 1424, Centre hospitalier Andrée Rosemon,

97300 Cayenne, Guyane française

G. Carles

Service de gynécologie obstétrique,

Centre hospitalier de l'Ouest guyanais,

Saint-Laurent-du-Maroni cedex, Guyane française

N. Villemant

Institut de géographie (UFR08),

Université Paris 1 Panthéon-Sorbonne University, Paris, France

D. Rousset

Centre nationale des arbovirus, laboratoire de virologie,

Institut Pasteur de la Guyane, Cayenne, Guyane française

E. Mosnier

Centres délocalisés de préventions et de soins,

Centre hospitalier Andrée Rosemon, Cayenne, Guyane française propres à la région amazonienne et latino-américaine. Nous discutons ici, sur les bases d'une revue de la littérature et de nos expériences de terrain, des enjeux et des stratégies à mettre en œuvre pour 2016 suite à l'épidémie de Zika dans notre territoire. Ces problématiques sont communes et généralisables en partie aux pays voisins.

Mots clés Virus Zika · Microcéphalie · Infections maternofotales $\cdot$ Infections sexuellement transmissibles $\cdot$ Guyane française $\cdot$ Amérique latine

Abstract Started in 2015 in Brazil, an outbreak linked to a little known arbovirus, Zika virus spread throughout Latin America. This virus, considered until recently as responsible of only mild symptoms, made mention of previously unsuspected complications, with severe neurological manifestations in adults and malformations of the central nervous system, including microcephaly, in newborns of mother infected during the pregnancy. While the continent is more accustomed to the succession of arbovirus epidemics, suspected complications and the many unknowns keys of the latter arriving raise many public health issues. French Guiana, a French territory located in the north-east of the continent, combines both European level of resources and climate and issues specific to the Amazon region and Latin America. We discuss here the issues for 2016 Zika virus epidemic in our region, many of them are generalizable to neighboring countries.

Keywords Zika virus $\cdot$ Microcephaly $\cdot$ Maternal-fetal infections · Sexually transmitted diseases $\cdot$ French Guiana Latin America

\section{Introduction}

Le virus Zika (ZIKV), est un arbovirus de la famille des Flaviviridae et du genre Flavivirus, comme le virus de la fièvre jaune, la dengue ou encore le West Nile. Il a été 
détecté pour la première fois en 1947 chez un singe macaque rhésus utilisé comme sentinelle lors d'une étude de la fièvre jaune dans la forêt Zika en Ouganda, puis chez l'homme 1952 en Ouganda suite à des études de séroprévalence, et chez un homme de 30 ans fébrile au Nigéria [15,16,33].

La transmission est essentiellement vectorielle par le biais de nombreuses espèces de moustiques, parmi lesquels Aedes aegypti, Aedes albopictus et Aedes polynesiensis [12]. Des cas de transmission sexuelle ont également été rapportés hors zones d'endémie, aux États-Unis, en France, au Chili et en Italie [20,30,51].

L'infection par le ZIKV était jusqu'en 2014 considérée comme bénigne associant de façon inconstante un syndrome éruptif, de la fièvre, des myalgies, des arthralgies et/ou une hyperhémie conjonctivale (figs 3, 4). Entre 1952, date de la première description chez l'humain, et la première épidémie en 2007, moins de 10 cas avaient été rapportés. Cette première épidémie, survenue sur l'île de Yap en Micronésie, a rapporté 185 cas suspects (dont 49 confirmés en PCR) et 59 probables (c'est-à-dire diagnostiqués par sérologie) tous bénins, sur une population de 7391 habitants [17]. Une étude de séroprévalence au décours de cette épidémie avait estimé à $73 \%$ la part de la population ayant été infectée par le virus, mettant en évidence un nombre important, environ $80 \%$, de cas a- ou pauci-symptomatiques. Cette réputation de bénignité a été récemment contredite lors de l'épidémie qui aurait touché environ 19000 personnes en Polynésie française en 2013-2014 [9]. Au décours de cette épidémie, 72 formes sévères à type de manifestations neurologiques graves dont 42 syndromes de Guillain-Barré (SGB), ainsi que des atteintes auto-immunes ont été associées à l'épidémie de ZIKV [9,40]. Le taux d'attaque du virus Zika en Polynésie française a été estimé à $66 \%$ [8]. L'épidémie s'est ensuite propagée à d'autres îles du Pacifique.

En mai 2015, le Brésil a déclaré des cas de Zika dans 7 États du Nord-Est. L'hypothèse retenue de l'arrivée du virus au Brésil est celle de l'importation par des athlètes ou des supporters en provenance du Pacifique, à l'occasion d'événements sportifs. Ainsi, un championnat de canoë auquel des athlètes de plusieurs îles du Pacifique touchées par l'épidémie participaient [7,21]. Une autre théorie implique des voyageurs (probablement des touristes plutôt que des sportifs) à l'occasion de la Coupe du monde de football de 2014 $[37,45]$. Depuis, le ministère de la Santé brésilien estime entre 500000 et 1,5 million le nombre de personnes qui auraient été infectées par le ZIKV dans la région du Nordeste en 2015. L'épidémie s'est ensuite progressivement répandue dans toute l'Amérique latine, touchant début mars 2016, 52 pays dans le monde dont 41 depuis le 1er janvier 2015 et 31 dans la zone dans les Amériques [56] (fig. 1).

En octobre 2015, les autorités de santé brésiliennes ont rapporté une augmentation du nombre de cas de microcéphalie chez les nouveau-nés dans certains Etats qui se trouvaient être ceux dans lesquels l'épidémie sévissait [50]. Depuis lors, plusieurs publications sont venues étayer l'association entre ZIKV et microcéphalie. Ainsi, deux cas de génome de ZIKV détectés dans le liquide amniotique ont été rapportés [47] et plusieurs cas de nouveau-nés avec microcéphalie décédés dans les premières heures de vie et 2 fœetus sur fausses couches spontanées (11 et 13 semaines d'aménorrhée SA) ont été testés positifs pour ZIKV [34]. Le tissu cérébral et le liquide amniotique d'un fotus issu d'une interruption thérapeutique à 32 semaines de grossesse pour hydrops fetalis (microcéphalie sévère, hydranencéphalie, calcifications intracrâniennes, lésions destructrices de la fosse postérieure, hydrothorax, ascite et œdème sous-cutané) ont également été testés positifs en PCR [46]. ZIKV a également été retrouvé en Slovénie dans le cerveau d'un fœetus avec malformations fotales à l'échographie au décours d'une autopsie, la mère ayant présenté un tableau compatible avec une infection par ZIKV au cours de sa grossesse au Brésil [36]. Début mars 2016, une équipe brésilienne a publié une étude dans le New England Journal of Medicine montrant que $12 \%$ de 42 femmes enceintes ayant eu une infection par ZIKV prouvée au cours de la grossesse présentaient des anomalies fœtales à l'échographie obstétricale [5]. Des chiffres non encore consolidés semblent montrer une incidence très élevée des fausses-couches chez les femmes ayant contracté l'infection par ZIKV au début de la grossesse à Saint Laurent du Maroni, à l'Ouest de la Guyane (Carles, données non publiées). Au même moment, une autre publication a montré que ZIKV infectait efficacement des cellules souches neurales humaines qui relarguaient secondairement des particules de ZIKV [49]. L'infection expérimentale de ces cellules par ZIKV provoquait une augmentation de la mort cellulaire, une dérégulation du cycle cellulaire à l'origine d'une croissance atténuée de ces cellules souches.

Bien que de nombreuses inconnues persistent, le lien possible entre microcéphalie chez les nouveau-nés et infection par ZIKV a provoqué une panique mondiale. Le 28 janvier 2016, la ministre française des Affaires sociales, de la Santé et des Droits des femmes appelait sur France Info les femmes enceintes de métropole à « différer leur voyage » en Guyane, en Martinique ou dans les territoires d'outre-mer en raison du ZIKV, recommandations qui ont été reprises ensuite par les sociétés savantes $[27,42]$. Outre le caractère exceptionnel de cette déclaration retentissante, l'absence de prise en compte initiale de la situation des femmes résidant en zone d'endémie a tout de suite marqué les esprits. Le lendemain, la ministre des Outre-mer, atténuait les propos de sa collègue en conseillant l'usage de répulsifs, de moustiquaires et de la climatisation aux femmes enceintes vivant en zone d'épidémie ou souhaitant s'y rendre [27].

Parallèlement, les gouvernements de plusieurs pays d'Amérique latine ont recommandé aux femmes en âge de procréer de différer leur grossesse de 6 mois à 2 ans [44]. De 


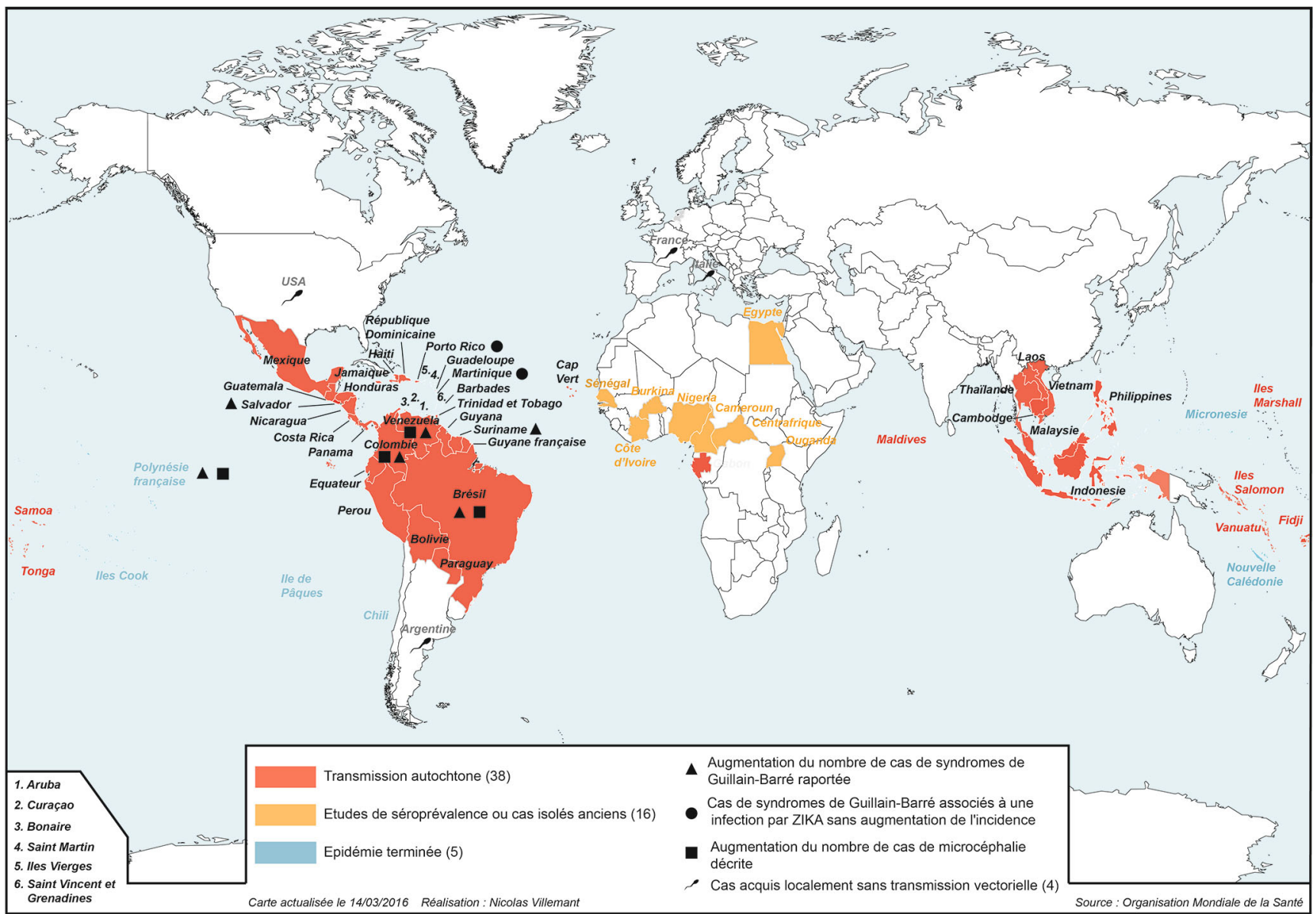

Fig. 1 Répartition des pays déclarant des cas de virus Zika en Amérique latine au 15 mars 2016 / Distribution of countries reporting cases of Zika virus in Latin America in March 15, 2016

son coté, le Haut Conseil de santé publique français (HCSP), comme l'OMS, a recommandé, fin janvier 2016, de spécialement veiller à ce que les femmes en âge de procréer et, plus particulièrement, les femmes enceintes aient les informations et le matériel nécessaires pour réduire le risque d'exposition [27].

\section{La situation en Guyane française}

La Guyane française est un département français d'outremer, situé en Amérique du Sud entre le Suriname et le Brésil. Le climat est de type équatorial et plus de $90 \%$ de sa surface (grande comme le Portugal) est recouverte de forêt amazonienne. Les $10 \%$ restants sont les zones côtières où se regroupent $90 \%$ des 250000 habitants (Insee 2013). Le département présente des caractéristiques sociodémographiques particulières : le taux de natalité le plus élevé de France après Mayotte, et l'un des plus élevé d'Amérique latine avec 6500 naissances par an, soit environ 2,6\% (fig 5). Elle est composée d'une population hétérogène avec de nombreuses communautés locales et $34 \%$ de la population est d'origine étrangère (Insee 2011), $20 \%$ vivant en dessous du seuil de pauvreté. De longue date, la Guyane française est familière avec les épidémies d'arboviroses : anciennement la fièvre jaune, les virus de la dengue et plus récemment le virus chikungunya.

Les premiers cas d'infection par le ZIKV ont été rapportés en décembre 2015 en Guyane, initialement des cas importés. La situation « épidémique » a été déclarée le 22 janvier 2016. Au 10 mars 2016, 1805 cas cliniquement évocateurs de ZIKV et 142 biologiquement confirmés ont été notifiés [11]. Une majorité des cas est signalée sur les secteurs du littoral, mais des cas ont également été identifiés dans les communes de l'intérieur sur le fleuve Maroni, qui sépare la Guyane du Suriname.

\section{Enjeux pour la recherche}

Il existe peu de littérature scientifique sur le ZIKV, qui est une maladie tropicale négligée. Ainsi, dans Pubmed, avec le 
mot clé « Zika », et en éliminant les chercheurs portant ce nom singulier, 262 références sont identifiées au $1^{\mathrm{er}}$ mars 2016 dont 127 (48,5\%) pour les seuls mois de janvier et février 2016. Ceci témoigne de l'augmentation brutale de l'intérêt porté à ce virus après les épidémies de Polynésie en 2013 et aujourd'hui dans le Nouveau monde. En comparaison, on retrouve 14900 et 2960 occurrences pour la « dengue » et le « chikungunya dans le même moteur de recherche (fig. 2).

\section{Quelles leçons tirer de l'expérience de la Polynésie française ?}

Les autorités de veille sanitaire ont bien été alertées par un nombre de cas anormalement élevé (18) de malformations congénitales cérébrales en 2014 au décours de l'épidémie de ZIKV, mais du fait d'une population trop restreinte, le lien de causalité n'a put être fortement suspecté qu'avec l'ampleur de l'épidémie brésilienne [6]. L'Institut Louis Malardé de Papeete a été très réactif et a permis d'apporter un grand nombre d'informations (PCR sur la salive, les urines, transmission par don de sang, séroprévalence, pourcentage d'asymptomatiques, etc.). Comme en Polyné- sie, la Guyane a la chance d'avoir un tissu de chercheurs compétents travaillant d'ores et déjà sur les arboviroses. Ainsi, une partie des connaissances actuelles sur le virus est issue des nombreuses publications concernant l'épidémie de 2014 par les chercheurs français en Polynésie, parfois en collaboration avec des équipes de métropole et internationales $[3,4,8,9,23,32,37,38,40]$. On remarquera qu'a contrario, pratiquement aucune donnée ni publication ne sont disponibles concernant l'épidémie qui a secondairement eu lieu en Nouvelle-Calédonie. L'objectif des chercheurs dans les départements français d'Amérique est d'être aussi réactif que leurs alter ego des îles françaises du Pacifique. Cela a déjà été le cas avec la publication par l'Institut Pasteur de Guyane du premier séquençage complet du génome du virus Zika américain, issu de prélèvements du Suriname [18]. En Guyane et aux Antilles françaises, il sera important de mettre en avant la recherche autour de ZIKV dans tous les domaines : épidémiologie, virologie, entomologie, etc., afin d'optimiser la prévention et la prise en charge des potentielles complications. Le challenge est donc de mobiliser toutes les compétences locales, de poser les bonnes questions scientifiques et de mettre en œuvre les moyens adéquats.

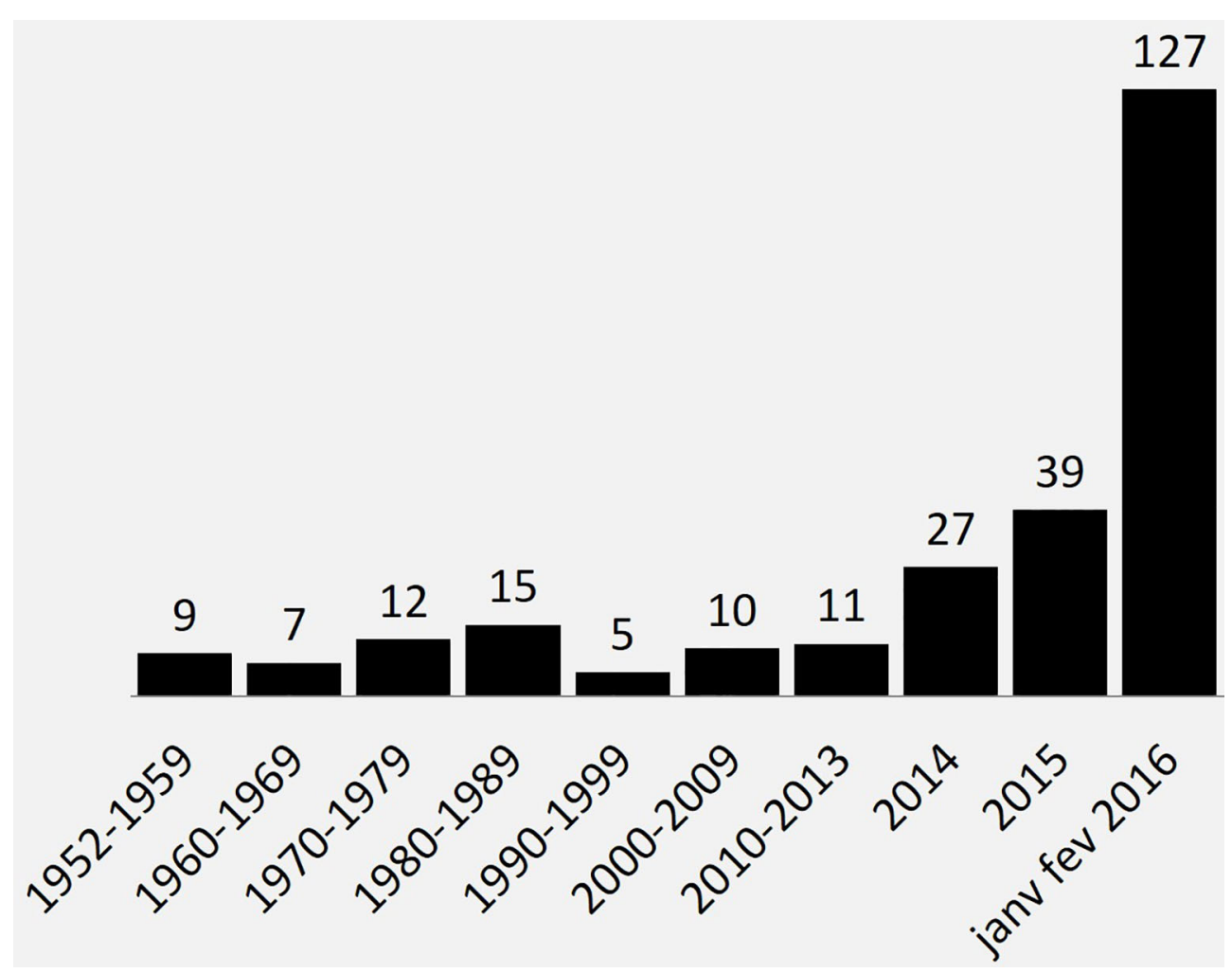

Fig. 2 Nombre de publications comprenant l'occurrence «Zika » dans Pubmed du 01/01/1952 - 29/02/2016 (après avoir exclu les auteurs nommés Zika, et 2 études dans la forêt Zika en Ouganda, sans lien avec ce virus) / Number of papers with "Zika » keyword in Pubmed of 01/01/1952 - 29/02/2016 (except authors named Zika, and 2 studies in the Zika forest, Uganda, with no relationship with the virus). 


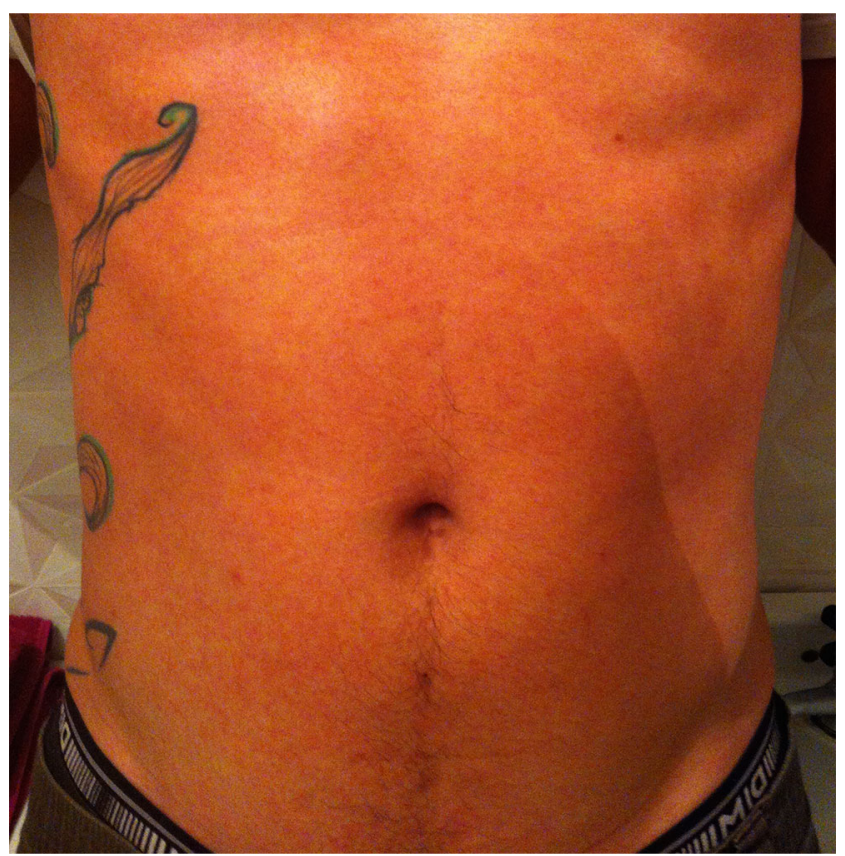

Fig. 3 Éruption cutanée au cours de l'infection par le virus Zika (Colombie, décembre 2015) / Rash during Zika virus infection (Colombia, December 2015).

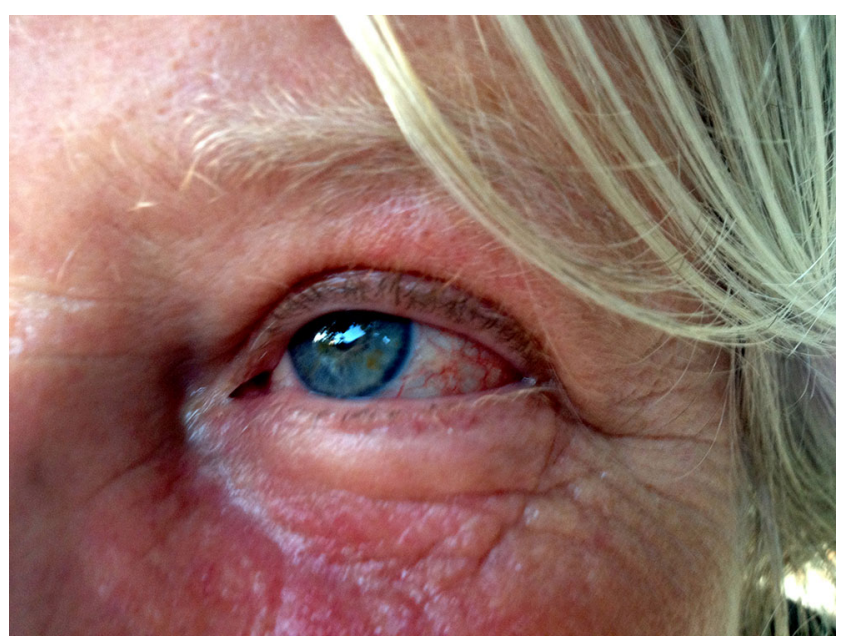

Fig. 4 Hyperhémie conjonctivale au cours de l'infection par virus Zika (Polynésie française, 2014) / Conjunctival injection during Zika virus infection (French Polynesia, 2014).

\section{Conséquences fotales d'une infection à ZIKV au cours de la grossesse}

C'est donc avec l'épidémie au Brésil que le lien de causalité entre les malformations neurologiques néonatales et l'infection par ZIKV a été fortement suspecté. Cette question est au cœur des débats actuels, soulevant des inquiétudes pour des millions de personnes vivant en zone d'endémie ou devant s'y rendre, avec des enjeux médicaux, politiques et économiques, à l'échelle régionale et internationale.

Des chiffres colossaux de microcéphalies ont été annoncés par les autorités de santé brésiliennes, mais après l'émotion initiale, des publications ont tempéré les premiers chiffres avancés, incitant à une plus grande prudence dans l'interprétation des données dans des régions où la veille sanitaire n'est pas toujours optimale [52]. Ainsi le ministère de la Santé brésilienne a publié le 2 mars 2016 une actualisation des chiffres, soit 5909 cas notifiés en 2015 et 2016 dont seulement 641 confirmés, 1046 écartés et 4222 en cours d'investigation [48]. Il est en effet indispensable de prendre en compte et d'écarter les autres causes d'anomalies neurologiques néonatales telles que les infections maternelles à cytomégalovirus (CMV), rubéole ou toxoplasmose, la consommation d'alcool, ou des maladies génétiques.

Dans le cas présent, la première difficulté est de définir précisément le facteur de risque, donc l'infection par ZIKV au cours de la grossesse. En effet, en cas d'infection symptomatique les patientes ne consultent pas toujours ou pas rapidement, or la virémie est courte, et la PCR (sang ou urines) n'est pas toujours disponible. Pour les 70 à $80 \%$ de gestantes asymptomatiques, la capacité des laboratoires en matière de sérologie n'est pas suffisante à ce jour pour envisager un dosage mensuel des anticorps, comme dans la toxoplasmose, pour rechercher une séroconversion. La deuxième difficulté est la définition de l'atteinte neurologique du fœtus : la définition de la microcéphalie n'est pas universelle et a changé ces derniers mois au Brésil, conduisant à des estimations très larges [52]. En outre, si la microcéphalie est la forme la plus grave d'atteinte fotale, elle semble être la partie émergée de l'iceberg des malformations cérébrales retrouvées, surtout en cas de contamination en début de grossesse, comme c'est le cas pour le CMV [14]. De nombreuses autres anomalies cérébrales ont été décrites : calcifications, ventriculomégalie, hypoplasie du corps calleux ou du cervelet, anomalies de la gyration, de l'artère cérébrale moyenne, kyste du plexus choroïde, agénésie du vermis, brachycéphalies [5,32]. D'autres types d'atteintes non neurologiques, oligohydramnios, insuffisance placentaire, hydrops fetalis et de nombreuses fausses couches spontanées ont également été rapportées [5]. Ainsi, de nombreuses questions restent incertaines malgré quelques éléments de réponse apportés par les études en cours : tous les trimestres de grossesse sont-ils à risque pour le fotus? Avec quel rapport de risque ? Les formes asymptomatiques de ZIKV chez une femme enceinte peuvent-elles provoquer des troubles neurologiques chez l'enfant? En l'absence de microcéphalie à la naissance, peut-il y avoir des anomalies de développement chez le nourrisson?

Dans le contexte guyanais, qui connait l'un des taux de natalité les plus importants du continent, le risque de foetopathie lié au ZIKV entraîne une crainte importante. Si l'on jongle avec les chiffres, on peut estimer, en considérant les 
ZIKA

\section{EPIDEMIE GUYANE}

\section{Etat des lieux}

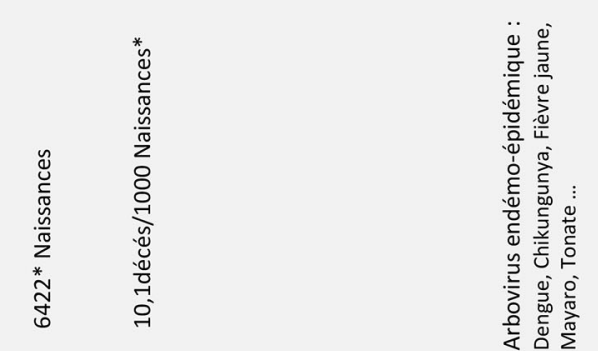

\section{००}
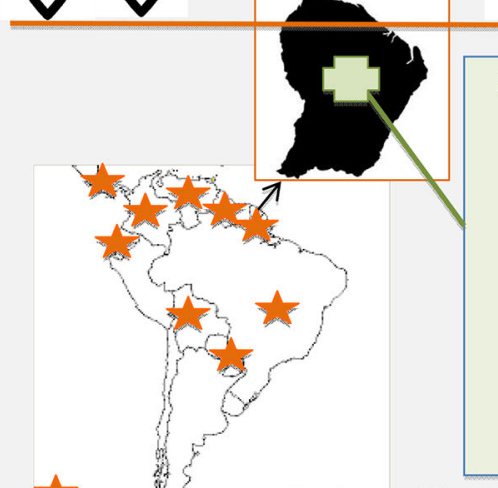

Transmission active de Zika virus***
Enjeux de recherche clinique

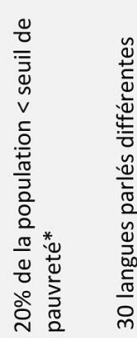

ZIKV

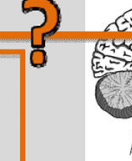

3 Hôpitaux
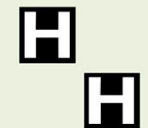

18
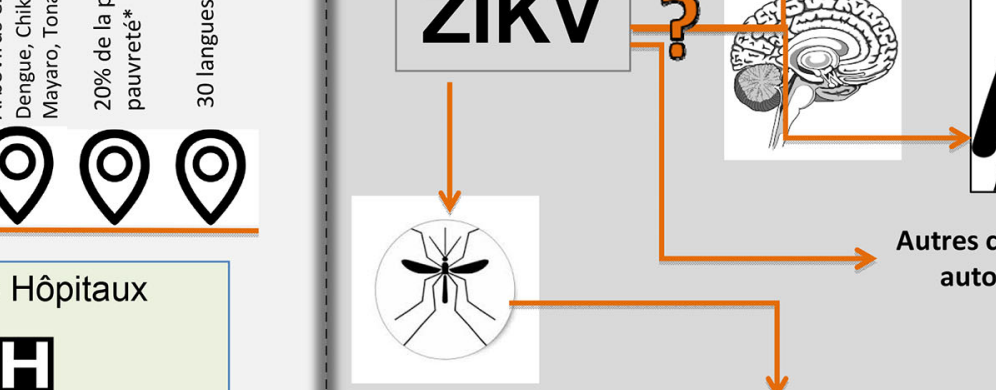

$\mathrm{SGB}^{1}$

Méningite

Encéphalite

Myélite

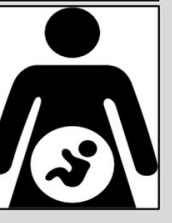

Autres complications auto-immunes
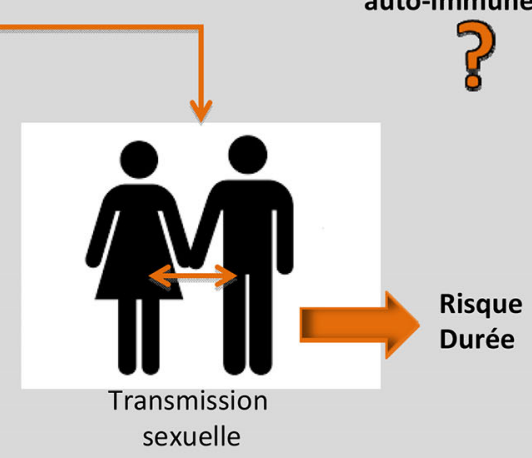

Risque Durée

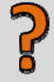

*Source INSSEE 2011-2012; **Centres Délocalisés de Prévention et de Soins; ${ }^{* * * C D C}$ 02/2016; 1 : Syndrome de Guillain Barré

Fig. 5 Infographie résumant les enjeux de l'épidémie de virus Zika en Guyane française / Figure summarizing the issues of the epidemic of Zika in French Guiana.

6500 grossesses actuelles rapportées par an et une incidence de $10 \%$ environ de ZIKV symptomatiques vs $70 \%$ d'asymptomatiques, que 650 à 4500 femmes enceintes seront infectées par le ZIKV. D'un autre coté, si l'on superpose l'estimation polynésienne de l'incidence des malformations fotales graves de 6 cas pour 1000 grossesses, on peut estimer la survenue d'environ 85 cas de fœtopathies graves en Guyane. Ces dernières estimations sont bien loin des chiffres évoqués par les confrères des pays voisins avec des bassins de population incomparablement plus grands. Cependant, le système de soin français en Guyane reste performant dans le contexte latino-américain, permettant la mise en place de cohortes et de collections biologiques. Des projets de recherches actuellement en cours de mise en place avec les Antilles permettront d'améliorer nos connaissances sur les conséquences fœtales de l'infection maternelle à ZIKV et la prise en charge des femmes enceintes dans le contexte d'épidémies à ZIKV, de diffuser des messages de prévention plus clairs, et de répondre à l'inquiétude de la population.

\section{Atteintes neurologiques}

En dehors des complications materno-fœtales évoquées plus haut, une augmentation importante de l'incidence de certaines atteintes neurologiques, comme les polyradiculonévrites aiguës, également appelées syndrome de Guillain-Barré (SGB), a été décrite de façon concomitante à la survenue d'épidémie de ZIKV (en Polynésie et sur le continent américain). Les informations tant attendues relatives à l'augmentation des cas de Guillain-Barré au cours de l'épidémie de ZIKV en Polynésie ont été publiées fin février 2016 [8]. Ainsi, 42 cas ont été rapportés lors de cette épidémie. Si $98 \%$ des patients avaient des IgM ou des IgG anti-ZIKV, et $100 \%$ en séroneutralisation, la PCR était négative pour tous. Le lien évoqué initialement avec les épidémies 
concomitantes par les virus de la dengue DENV \& 1 et 3 a pu être écarté à cette occasion. D'autres pays ont rapporté leurs premiers cas de syndrome de Guillain Barré lié au ZIKV en 2016 : Colombie, Salvador, Venezuela, Martinique, Puerto Rico, Panama, Surinam [56], et à l'heure où ces lignes sont écrites, 3 ont été suspectés en Guyane parmi lesquels un cas a été exclu, un a présenté une sérologie ZIKV positive en IgM et un en cours d'investigation [11]. D'autres anomalies neurologiques ont été rapportées en Polynésie (méningite et méningo-encéphalite). En confirmation de ce neurotropisme et de cette neurovirulence directe, un cas de méningo-encéphalite avec culture et recherche PCR positive dans le LCR de ZIKV vient d'être rapporté chez un homme de 81 ans au retour d'un voyage dans le Pacifique et un cas de myélite attribuée au ZIKV a également été rapporté début mars 2016 en Guadeloupe [10,35]. Des complications hématologiques, thrombopénies ou anémies auto-immunes ont également été évoquées en Polynésie dont le détail n'est pas encore publié. En pratique, en Guyane, il faut évoquer ZIKV lors de tout diagnostic de maladie du système nerveux central, avec un bilan standardisé et un recueil prospectif des données, et rester particulièrement vigilant face à toute pathologie atypique de nature neurologique, hématologique et auto-immune sans explication claire et dont le lien avec l'infection par ZIKV devra être recherché. L'enjeu ici est de rester vigilant afin de mettre en évidence ce que l'on ne connaît pas encore et de « cocher » la case « atteinte sévère » permettant la recherche du virus devant un tableau non documenté de pathologie d'allure infectieuse inhabituelle ou grave. Dans ce contexte, la création d'un centre de ressource biologique est l'un des projets phares du centre hospitalier de Cayenne pour 2016.

\section{Enjeux diagnostics}

De la surveillance clinique d'un syndrome évoquant une infection à arbovirus au séquençage complet du génome [18], il y a une grande distance. Comme toujours au cours des épidémies d'arbovirose, on se demande jusqu'à quel moment et dans quelles populations on doit confirmer biologiquement le diagnostic du ZIKV. En Guyane française, ceci a été vécu de façon un peu étrange par les médecins et la population. En effet, alors que les annonces de l'apparition de ZIKV dans différents pays d'Amérique du Sud se multipliaient, aucun cas n'était identifié en Guyane. Début janvier, peu de temps après le diagnostic des premiers cas autochtones, la répartition des cas d'emblée confirmés sur l'ensemble du territoire du littoral - traduisant probablement une circulation à bas bruit -, a incité les autorités sanitaires à déclarer rapidement le stade " épidémie ». Cette situation originale a été celle d'une déclaration de phase épidémique alors que peu de patients étaient vus en consultation en ville ou à l'hôpital pour ce motif. Ceci est expliqué par le fait que cette arbovirose est peu symptomatique et surtout que les populations guyanaises connaissent bien les infections arbovirales. Cette infection est donc peu médicalisée entraînant ce décalage entre déclaration d'épidémie et peu de cas reçus en consultation, ce qui avait déjà été notifié en Polynésie française [8]. Il n'existe pas à proprement parler de réseau sentinelle de médecins généralistes en Guyane.

Mi-février 2016, le diagnostic biologique est réservé aux femmes enceintes avec suspicion de ZIKV, aux nouveau-nés avec malformations, ainsi qu'aux cas jugés sévères en milieu hospitalier. Le diagnostic actuel à la mi-mars repose sur une PCR qui peut être réalisée dans le sang jusqu'à $\mathrm{J} 5$ après le début des symptômes et dans les urines jusqu'à J10, comme l'a reprécisé récemment la saisine de la Haute autorité de santé [29]. Jusqu'à fin février, le diagnostic virologique se faisait exclusivement à l'Institut Pasteur de Cayenne, et dans quelques laboratoires de virologie en métropole, mais des kits commercialisés par la firme Altona Diagnostics sont désormais disponibles au laboratoire de l'hôpital de Cayenne. Du fait d'un nombre de commandes brutal et massif, les capacités $\mathrm{du}$ laboratoire pharmaceutique qui les fabrique risquent d'être dépassées. Quant à la mise en place de la sérologie, des kits commerciaux seront bientôt en cours d'évaluation à l'Institut Pasteur de Cayenne qui a mis en place récemment la technique maison. La prudence dans l'interprétation des résultats sera de mise puisque d'autres flavivirus circulent dans le département comme la dengue, et d'autres dans la région (virus de l'encéphalite de Saint Louis, West Nile virus, Ilheus virus, etc.), sans compter la vaccination systématique contre la fièvre jaune, et des fausses sérologies dengue et/ou flavivirus tout venant qui risquent de perturber l'interprétation de certaines sérologies ZIKV positives.

\section{Enjeux de la prévention}

Dans les médias, l'exigence de mise à disposition immédiate d'un vaccin est soulevée, comme à chaque épidémie. Il est cependant fort probable que, au vu des délais de mise au point de ces vaccins, celui-ci ne soit disponible que lorsque l'épidémie sera finie. Ainsi, l'OMS annonçait le 12 février 2016 la possibilité de premiers essais d'ici 18 mois, après avoir sélectionné 2 entreprises suffisamment avancées dans la mise au point de vaccins basées sur d'autres vaccins contre d'autres flavivirus (encéphalite japonaise, dengue). Aussi, les messages de prévention sont basés sur la prophylaxie d'exposition et les recommandations à l'endroit des femmes enceintes ou en âge de procréer. S'il est relativement aisé de recommander à celles qui vivent hors des zones épidémiques de ne pas se rendre dans les régions où sévit ZIKV, l'enjeu est beaucoup plus complexe pour les résidentes [41]. En effet, que conseiller, en zone d'endémie, en cas de désir de grossesse ? Ainsi l'une des principales recommandations 
émises notamment par le HCSP est le report du projet de grossesse [27] en pariant sur la «non-endémisation » du virus ZIKV et/ou sur la rapide décroissance des cas, voire sur l'immunité acquise de la mère après la phase épidémique. La durée courte de l'épidémie dans les îles du Pacifique touchées par le virus Zika en 2013-2014 permet de croire à une évolution dans ce sens en Amérique latine, quoique la situation géographique continentale et la densité de population puissent rendre plus aléatoires ces prévisions. Cependant, vu depuis l'Amérique latine, ces recommandations seront peut-être difficiles à appliquer. En effet, comme le souligne une lettre publiée par le Lancet en février 2016, $56 \%$ des grossesses en Amérique latine ne sont pas désirées, et l'accès à l'éducation, à la contraception, la prévalence élevée des viols et les barrières culturelles rajoutent une difficulté supplémentaire à l'applicabilité de ces recommandations [44]. Le HCSP recommande pour les femmes enceintes ou ayant un projet de grossesse ainsi que pour les femmes en âge de procréer, vivant dans les zones touchées par une épidémie de virus Zika, une information sur les embryofœthopathies et autres complications pouvant survenir lors d'une infection par le virus Zika ; une sensibilisation sur l'intérêt du respect, par l'ensemble de la population des mesures de lutte et de prévention personnelle antivectorielles et une incitation à consulter un médecin afin de définir les mesures de prévention les plus adaptées à leur situation, notamment en cas de signes cliniques évocateurs d'une infection Zika [27]. La question de l'information à délivrer est complexe, car à ce jour nous manquons encore d'éléments et d'outils pédagogiques adaptés. Choisir la prudence et l'information éclairée du patient semble être la meilleure des solutions, même si, compte tenu de l'état actuel de nos connaissances, la réponse à donner à un couple ayant un projet de grossesse aux questions posées concernant les risques d'une infection par le ZIKV ne peut être que parcellaire. Ces interrogations reviennent d'ailleurs déjà quotidiennement auprès des équipes soignantes et il serait dangereux de les éluder. Enfin, d'autres questions sont en cours d'exploration, notamment celles de l'allaitement en cas d'infection au virus Zika dont l'OMS vient de recommander la poursuite et celle de la prise en charge psycho-sociale des familles dont l'enfant est atteint de microcéphalie [53, 54].

Pour ce qui est du suivi des grossesses, le ministère des Affaires sociales et de la Santé, dans un courrier daté du 24 février 2016, a recommandé à l'Assurance maladie et à l'Assurance maternité de prendre en charge les échographies obstétricales des $18^{\mathrm{e}}, 28^{\mathrm{e}}$ et $36^{\mathrm{e}}$ semaines d'aménorrhée (SA), en plus des 3 échographies habituellement requises $\left(12^{\mathrm{e}}, 22^{\mathrm{e}}\right.$ et $\left.32^{\mathrm{e}} \mathrm{SA}\right)$ pour les femmes assurées enceintes exposées à un risque d'infection par le virus Zika. Si cette directive est accueillie avec intérêt par les professionnels de santé, son application dans le contexte guyanais interroge. En effet, dans notre département, seules $40 \%$ des patientes effectuent les 3 examens recommandés, et les capacités de réalisation de cet examen requérant une expérience, un savoir-faire, et un temps d'examen importants. Si la Guyane semble disposer d'un nombre d'échographes suffisant, au moins au $\mathrm{CH}$ de Cayenne, le nombre d'échographistes de niveau 2 n'est probablement pas suffisant à l'heure actuelle [13]. L'Établissement de préparation et de réponse aux urgences sanitaires (EPRUS) a dans ce sens proposé un renfort en termes d'échographistes dans les mois à venir. En outre, si une majorité de la population guyanaise vit sur le littoral, celle des communes isolées sur les fleuves Maroni et Oyapock, avec de forts taux de natalité, représente un enjeu supplémentaire, car il n'y a pas d'obstétriciens sur place, et les missions spécialisées devront être multipliées pour répondre à ce défi. Dans le contexte actuel, une organisation spécifique devra être pensée pour l'organisation dans les Centres délocalisés de prévention et de soins (CDPS) de ces suivis échographiques. Pour exemple et en ce sens, une web-formation sur les signes échographiques à rechercher, réalisée par l'université du Colorado et soutenue par l'OMS et l'Organisation panaméricaine de la Santé (OPS) a été mise à disposition à l'attention des médecins des sites isolés.

\section{Quid de la transmission sexuelle ?}

Plusieurs cas de transmission sexuelle probable de ZIKV ont été rapportés aux Etats-Unis, en France, en Italie, et probablement en Argentine et au Canada [20,30,51]. On a également rapporté le cas d'un homme présentant une hémospermie sur prostatite avec excrétion de virus Zika dans le sperme [39]. Dans le cas de ce patient, on pouvait considérer le patient infectieux, puisque le sperme n'était pas seulement positif en PCR mais aussi en culture. Une autre publication du mois de février a rapporté la persistance d'une excrétion de ZIKV dans le sperme, plus de 2 mois après la guérison chez un homme de 68 ans [2]. En conséquence, le Center of Diseases Control nord-américain et le HCSP recommandent aux hommes résidant ou ayant voyagé dans une zone de transmission active du ZIKV, l'abstinence ou le port du préservatif quel que soit le type de rapport, que leur partenaire soit enceinte ou non, pour une durée indéterminée [28]. Ces recommandations, qui semblent relever du bon sens, appellent cependant de nombreux questionnements concernant leur applicabilité sur le terrain. Malgré la peur de la microcéphalie, on peut douter que les populations locales suivent assidument les recommandations de prévention de la transmission du ZIKV, en raison, entre autres, des facteurs socioculturels et religieux.

\section{Accès à la contraception et à l'avortement : un défi pour certaines communautés}

Même si l'accès à la contraception et à l'avortement se doit, théoriquement d'être ouvert et accessible à toutes les 
femmes sur le territoire français, cela n'est malheureusement pas toujours simple en Guyane française. Ainsi, du fait d'un isolement géographique important, les communautés vivant sur les fleuves ont un accès plus difficile à l'avortement médicamenteux. Par ailleurs, en raison du coût élevé de certains moyens de contraception comme le stérilet ou d'un manque d'information, les femmes vivant en zones isolées ou les femmes précaires du littoral n'ont que peu de choix quant à leur mode de contraception. L'épidémie de ZIKV dans ce contexte risque donc d'aggraver l'inégalité dont elles sont déjà victimes.

D'autre part, les niveaux scolaires, notamment en zones isolées, sont bas et corrélés à l'âge de la première grossesse et du nombre d'enfants, reflétant des messages d'éducation à la santé qui ne passent déjà pas dans certaines communautés. Le nombre de grossesses précoces y est globalement le plus élevé de France (Insee 2011). Enfin, comme dans toute communauté, l'importance de ces grossesses par la force symbolique et émotionnelle, familiale et collective dont elles sont porteuses va devoir nous faire réfléchir à un discours adapté de prévention. Pour ce faire l'aide et l'implication des communautés via des médiateurs de santé, mais aussi la création d'outils de prévention en langue vernaculaire paraissent indispensables et urgentes.

\section{Lutte anti-vectorielle : une impasse scientifique?}

Se protéger contre les moustiques, quoi de plus difficile à faire passer comme message en zone tropicale ! En effet les populations vivent toute l'année avec les moustiques. La lutte anti-vectorielle repose à la fois sur des mesures individuelles et collectives. À l'échelle individuelle, deux principaux outils sont mis en avant : les répulsifs individuels et les moustiquaires. Si les répulsifs individuels sont plutôt bien acceptés et utilisés par une frange de population plutôt favorisée, leur coût prohibitif empêche leur utilisation à une large échelle. De plus, certains répulsifs sont contre-indiqués chez les femmes enceintes et ceux autorisés ne sont pas forcément les plus efficaces. Au niveau des autorités sanitaires guyanaises et antillaises, il a bien été envisagé de distribuer ces produits larga manu aux populations, mais le risque de mésusage et de dérive de cette distribution de masse a finalement écarté la possibilité d'une telle mesure. Les moustiquaires imprégnées font certes partie des outils prônés pour la lutte contre les piqures d'Aedes [27], mais il faut rappeler d'emblée que les moustiques vecteurs du ZIKV, mais aussi de la dengue, du chikungunya et de la fièvre jaune, Aedes aegypti ici, et ailleurs Aedes albopictus et Aedes polynesiensis, sont des moustiques majoritairement diurnes. Contrairement à ce qui se passe dans la lutte contre les anophèles vecteurs du paludisme qui sont des moustiques ayant une activité nocturne, l'usage des moustiquaires n'a donc, dans le cas des Aedes, qu'un intérêt limité. Elles peuvent bien entendu être proposées aux personnes alitées, en particulier les femmes enceintes, aux enfants avant l'âge de la marche et aux personnes atteintes par le ZIKV ou d'une autre arbovirose, mais cela représente une infime partie de la population concernée. Il existe dans une moindre mesure un effet insecticide qui est censé agir sur les moustiques diurnes comme nocturnes par l'effet « knock down », c'est-à-dire une paralysie des muscles et du système nerveux des insectes entrâ̂nant leur mort, suite au contact avec un insecticide du type pyréthrinoïde [31]. Une situation quasi cornélienne s'est présentée dans les régions équatoriales françaises concernant les moustiquaires imprégnées qui ont montré une efficacité infiniment supérieure à celles qui ne le sont pas, mais qui ont l'inconvénient de faire appel à des produits chimiques soumis à réglementation. Jusqu'ici, l'insecticide utilisé dans les départements français d'Amérique était la deltaméthrine qui s'est vue récemment retirer son autorisation de mise sur le marché dans l'Union européenne rendant ainsi des milliers de moustiquaires disponibles, inutilisables. Celles qui doivent les remplacer, imprégnées à la perméthrine et fabriquées en Allemagne, ne seront pas disponibles avant plusieurs semaines et utilisables tout au plus jusqu'en juillet 2016, date à laquelle elles seront elles aussi interdites. Cela laisserait les départements français d'Amérique sans autre choix que l'utilisation de moustiquaires non imprégnées infiniment moins efficaces. Le HCSP a donc recommandé par un avis émis le 18 janvier 2016 que l'autorité compétente française auprès de l'Union européenne fasse une demande de dérogation pour l'utilisation des moustiquaires imprégnées à la deltaméthrine [26]. Une dérogation a finalement été obtenue par l'Agence régionale de santé de Guyane qui autorise les femmes enceintes uniquement à utiliser les moustiquaires imprégnées de deltaméthrine distribuées dans les CDPS.

La lutte contre Aedes sp., qui est un moustique majoritairement à faible périmètre de vol (quelques dizaines à centaines de mètres maximum) passe par l'éradication des gîtes domestiques. Or, si sur le papier cela semble raisonnable, la pratique quotidienne est tout autre. En effet, en zone tropicale, pendant la saison des pluies, les flaques et autres points d'eau s'accumulent. Si nettoyer ses gouttières et mettre du sable dans les coupelles de pots de fleurs restent de l'ordre du possible, d'autres difficultés existent. En effet, la plupart des maisons sont équipées de réservoir recueillant les eaux de pluie, qui sont rarement couverts d'une moustiquaire et où prolifèrent les larves d'Aedes. En outre, une partie de la lutte contre ces gîtes passe par des mesures nécessitant un investissement des communes, ce qui n'est pas toujours le cas : lutte contre les décharges sauvages, élimination des véhicules hors d'usage, lutte contre les stagnations d'eau liées à certains défauts de construction. Enfin, les grandes villes de Guyane ont leur lot de quartiers plus ou moins illégaux où prolifèrent les moustiques. En effet, certains quartiers reculés, bâtis à la 
va-vite par des populations majoritairement clandestines n'ont pas pu être viabilisés et n'ont, de ce fait, pas d'accès à l'eau courante, ni électricité, ni ramassage des ordures, ni systèmes d'assainissement aux normes. L'épidémie de chikungunya qui a précédé celle du ZIKV a initialement flambé à Cayenne dans ces quartiers les plus défavorisés et les plus difficiles d'accès aux moyens de lutte anti-vectorielle [19]. Enfin, reste la grande polémique de la démoustication collective, qui a tant échauffé les esprits ces dernières années, notamment lors de l'épidémie de virus chikungunya de 2014-2015. En effet, entre toxicité et résistance, peu de produits restent actuellement utilisables. Ainsi après la disparition, entre autres, du DDT, le malathion a fait l'objet d'une polémique en 2014-2015 : interdit en Europe depuis 2007 et en France depuis 2008, autorisé à titre dérogatoire et sous de multiples conditions en Guyane par le HCSP en avril 2015, pour être finalement interdit quelques semaines plus tard en raison du risque cancérigène [24,25]. De plus, les études récentes montrent une forte résistance des vecteurs à la deltaméthrine et au malathion [22].

\section{Risque transfusionnel ?}

Du fait de l'endémicité de la maladie de Chagas et du risque de transmission par transfusion, il n'y a pas en Guyane de dons de sang et donc pas de risque transfusionnel à prévoir [38]. Cependant la question reste cruciale concernant les personnes de retour, en Europe, d'Amérique latine.

\section{De la logistique et de l'organisation à tous les niveaux}

La Guyane est un territoire continental où le réseau routier n'existe que sur le littoral, le reste du territoire n'étant accessible que par pirogue, avion et hélicoptère. Dans ce contexte, il va falloir s'adapter à la situation créée par l'épidémie de Zika, à savoir les outils de télémédecine, la formation des soignants, mais aussi les capacités de rapatriement, voire d'hospitalisation des femmes enceintes vivant sur le fleuve et ne pouvant, du fait de leur isolement, bénéficier d'un suivi selon les recommandations.

De plus, l'augmentation du nombre de cas de SGB, neuropathies nécessitant des durées souvent longues d'hospitalisation en réanimation, fait craindre un débordement des capacités d'accueil de ce service. En effet, en Guyane, il n'existe qu'un seul service de réanimation avec 12 lits pour 250000 habitants. Il faut réfléchir à augmenter le nombre de lits de réanimation, mais aussi de personnels et de matériel nécessaires à leur fonctionnement. Enfin, les dotations en immunoglobulines (traitement de première ligne recommandé pour les SGB) doivent être adaptées. La solution qui a été proposée est une alerte en cas de nécessité d'occupation des lits de réanimation au-delà de 15 patients, et d'évacuation sanitaire au-delà de 18 . Il faut aussi s'interroger sur la capacité des filières en aval dans les services de médecine, notamment la neurologie, de soins de suite et de rééducation qui sont peu nombreux en Guyane, afin d'éviter un engorgement rapide des hôpitaux. De même un scénario catastrophiste doit prendre en compte l'augmentation importante du nombre de nouveau-nés avec des malformations neurologiques, justifiant d'optimiser les services de néonatologie pour l'arrivée de ces enfants notamment en matériel et en personnel. Ces mesures doivent êtres pensées dès à présent en coordination avec l'ensemble des réseaux départementaux de soins de Guyane et des Antilles françaises. Enfin, les évacuations sanitaires se feront probablement prioritairement vers la métropole au vu du risque de saturation des hôpitaux antillais, et ceux de Martinique en particulier, qui subissent également l'épidémie de plein fouet.

\section{Informer : de la communication globale à l'information ciblée}

Comme lors de chaque épidémie, des rumeurs et des informations contradictoires n'ont pas tardé à se répandre sur les causes et les raisons de cette épidémie ainsi que sur l'origine de l'augmentation des cas de microcéphalie. Depuis quelques semaines des théories, dont il est difficile de dire si elles sont totalement farfelues ou reposent sur des arguments qui doivent être pris en compte circulent : rôle de moustiques transgéniques relargués dans le nord-est brésilien pour lutter contre les arboviroses, rôle de lots de vaccins défectueux contre la rubéole, épandage massif de produits phytosanitaires et herbicides dans des plantations industrielles de la même région comme le glyphosate, des insecticides et des larvicides, comme le dernier incriminé, le pyriproxyfène, qui aurait été versé dans les canalisations d'eaux pour lutter contre les moustique [43]. Tout ceci contribue à « flouter » les messages délivrés par les institutions et les soignants. Le défi est ici de délivrer une information adaptée à chaque personne et population impactée, répondant au mieux aux questions légitimes sur le « pourquoi » de cette épidémie et sur le « comment » en prévenir les éventuelles complications. À ce titre, l'OMS, la Croix rouge et l'Unicef viennent de publier des recommandations sur les messages clés à délivrer, des outils pédagogiques, mais surtout sur les principes de caractère participatif que doivent avoir ces campagnes d'information auprès des communautés [55].

\section{Et après le pic, quelle prise en charge au long cours ? Ou la peur d'être oubliés}

En Guyane française, si les conséquences tératogènes du ZIKV se confirment, les structures d'accueil d'enfants et d'adultes handicapés seront insuffisantes, car il existe un retard structurel important par rapport à la situation présente dans l'hexagone [1]. C'est là, peut être le plus gros enjeu de 
santé publique à moyen et long terme, comme cela a été relevé au Brésil, mais aussi par l'ONU qui n'a pas hésité à se positionner pour réclamer l'accès à la contraception. Ces besoins au long cours pourraient être importants, et nécessiteraient donc des mesures d'aide financière pérennes ce qui est l'un des points les plus difficiles à assurer dans la gestion des épidémies.

\section{Conclusion}

De nombreuses questions restent encore sans réponse, les connaissances scientifiques de ce virus et de ces conséquences pathologiques sur l'homme étant encore extrêmement parcellaires. L'enjeu est ici de rester ouverts et vigilants à d'autres complications possibles. La question difficile posée à la veille sanitaire à l'échelle locale mais aussi mondiale est de repérer ce que l'on ne connaît pas encore. Cependant plusieurs problématiques majeures inhérentes à cette épidémie dans le contexte guyanais ont déjà été identifiées : diagnostiquer et suivre médicalement les femmes contractant un ZIKV au cours de la grossesse, même celles vivant à l'écart du système de soins (géographiquement ou socialement) ; informer avec des messages adaptés les populations et mettre à disposition des moyens de prévention (moustiquaires, contraception, etc.) ; anticiper une surcharge de patients dans les hôpitaux, notamment en réanimation, mais aussi dans les services de gynécologie-obstétrique et de néonatologie ; enfin, prendre en charge au long cours les enfants qui auraient des séquelles neurologiques suite à cette épidémie.

Il sera nécessaire de partager l'expérience de terrain de gestion de cette épidémie ainsi que les données scientifiques qui pourront être recueillies afin de valoriser l'expérience guyanaise (fig. 5).

Liens d'intérêts : les auteurs déclarent ne pas avoir de liens d'intérêts.

\section{Références}

1. Agence Régionale de Santé de Guyane (2010) Enquête «ES Handicap ». Etablissements de santé pour personnes handicapées, $12 \mathrm{pp}$

2. Atkinson B, Hearn P, Afrough B, et al (2016) Detection of Zika Virus in Semen. Emerging Infectious Disease journal 22(5)

3. Aubry M, Finke J, Teissier A, et al (2015) Seroprevalence of arboviruses among blood donors in French Polynesia, 20112013. Int J Infect Dis 41:11-2

4. Baronti C, Piorkowski G, Charrel RN, et al (2014) Complete coding sequence of zika virus from a French polynesia outbreak in 2013. Genome Announc 2(3)

5. Brasil P, Pereira JP Jr, Raja Gabaglia C, et al (2016) Zika Virus Infection in Pregnant Women in Rio de Janeiro - Preliminary Report. N Engl J Med [Epub ahead of print]
6. Bureau de Veille Sanitaire et Centre Hospitalier de Polynésie française (2015) Note sur les investigations autour des malformations cérébrales congénitales ayant suivi l'épidémie de zika de 2013-2014 2 décembre 2015 [http://www.hygiene-publique.gov. $\mathrm{pf} / \mathrm{IMG} / \mathrm{pdf} /$ note_malformations_congenitales_cerebrales.pdf]

7. Campos GS, Bandeira AC, Sardi SI (2015) Zika Virus Outbreak, Bahia, Brazil. Emerg Infect Dis21(10):1885-6.

8. Cao-Lormeau VM, Blake A, Mons S, et al (2016) Guillain-Barre Syndrome outbreak associated with Zika virus infection in French Polynesia: a case-control study. Lancet S0140-6736(16)00562-6

9. Cao-Lormeau VM, Roche C, Teissier A, et al (2014) Zika virus, French polynesia, South pacific, 2013. Emerg Infect Dis 20 (6): $1085-6$

10. Carteaux G, Maquart M, Bedet A, et al (2016) Zika Virus Associated with Meningoencephalitis. N Engl J Med [Epub ahead of print]

11. Cellule interrégionale d'Epidémiologie (CIRE) Antilles-Guyane (2016) Emergence du virus Zika aux Antilles Guyane - Situation épidémiologique - Point épidémiologique du 10 mars. Le Point épidémio, $8 \mathrm{pp}$

12. Chouin-Carneiro T, Vega-Rua A, Vazeille M, et al (2016) Differential Susceptibilities of Aedes aegypti and Aedes albopictus from the Americas to Zika Virus. PLoS Negl Trop Dis 10(3): e0004543.

13. Conseil National Professionnel de Gynécologie et Obstétrique (2016) Virus ZIKA et femme enceinte ou en âge de procréer Document d'information temporaire, à l'intention des professionnels de santé, Version 1.3 du 3 février 2016 susceptible de mise à jour en temps réel:68 pp

14. Delay F, Coste Burel M, Joubert M, Winer N (2016) Infection materno-fœtale à CMV : étude rétrospective dans un CPDPN sur une période de 14 ans. J Gynecol Obstet Biol Reprod (Paris) S0368-2315(15)00389-0

15. Dick GW (1953) Epidemiological notes on some viruses isolated in Uganda; Yellow fever, Rift Valley fever, Bwamba fever, West Nile, Mengo, Semliki forest, Bunyamwera, Ntaya, Uganda S and Zika viruses. Trans R Soc Trop Med Hyg 47(1):13-48

16. Dick GW, Kitchen SF, Haddow AJ (1952) Zika virus. I. Isolations and serological specificity. Trans R Soc Trop Med Hyg 46 (5):509-20

17. Duffy MR, Chen TH, Hancock WT, et al (2009) Zika virus outbreak on Yap Island, Federated States of Micronesia. N Engl J Med 360(24):2536-43

18. Enfissi A, Codrington J, Roosblad J, et al (2016) Zika virus genome from the Americas. Lancet 387(10015):227-8

19. Epelboin L, Bonifay T, Bidaud B, et al (2015) Chikungunya en Guyane : une maladie des quartiers défavorisés ? $4{ }^{\mathrm{es}}$ Journées Interrégionales de veille sanitaire des Antilles Guyane. BVS 89:19-20

20. Foy BD, Kobylinski KC, Chilson Foy JL, et al (2011) Probable non-vector-borne transmission of Zika virus, Colorado, USA. Emerg Infect Dis 17(5):880-2

21. Gautret P, Simon F (2015) Dengue, chikungunya and Zika and mass gatherings: What happened in Brazil, 2014. Travel Med Infect Dis 14(1):7-8

22. Girod R, Gaborit P, Carinci R, et al (2015) Evaluation de la sensibilité au malathion et à la deltaméthrine des populations d'Aedes aegypti de Guyane. $4^{\mathrm{e}}$ Journées Interrégionales de veille sanitaire des Antilles Guyane. BVS 8-9:18

23. Hancock WT, Marfel M, Bel M (2014) Zika virus, French Polynesia, South Pacific, 2013. Emerg Infect Dis 20(11):1960

24. Haut Conseil de la santé publique (2014) Avis relatif aux conditions d'utilisation et aux mesures de gestion à mettre en place pour l'emploi dérogatoire du malathion en Guyane pour la lutte anti-vectorielle - complément d'information sur les mesures de protection pour les piscines - 13 novembre 2014,2 pp 
25. Haut Conseil de la santé publique (2015) Avis - Lutte contre les vecteurs du virus responsables du Chikungunya en Guyane après le classement du malathion comme cancérogène probable par le Centre international de recherche sur le cancer - Autosaisine 9 avril 2015, 3 pp

26. Haut Conseil de la santé publique (2016) Avis relatif aux mesures de gestion pour l'utilisation de la deltaméthrine dans les départements et territoires français d'Amérique -18 janvier 2016, $10 \mathrm{pp}$

27. Haut Conseil de la santé publique (2016) Avis relatif à l'actualisation de l'avis du HCSP du 28 juillet 2015. Avis relatif à la prise en charge médicale des personnes atteintes par le virus Zika 20 janvier 2016, $17 \mathrm{pp}$

25. Haut Conseil de la santé publique (2016) Avis relatif à la transmission du virus Zika par voie sexuelle -8 février 2016, 5 pp

29. Haute Autorité de Santé (HAS) (2016) Détection par RT-PCR du virus Zika dans le sang et les urines. Mars 2016, 33 pp

30. Hills SL, Russell K, Hennessey M, et al (2016) Transmission of Zika Virus Through Sexual Contact with Travelers to Areas of Ongoing Transmission - Continental United States, 2016. MMWR Morb Mortal Wkly Rep 65(8):215-6

31. Hougard JM, Duchon S, Darriet F, et al (2003) Comparative performances, under laboratory conditions, of seven pyrethroid insecticides used for impregnation of mosquito nets. Bull World Health Organ 81(5):324-33

32. Jouannic JM, Friszer S, Leparc-Goffart I, et al (2016) Zika virus infection in French Polynesia. Lancet S0140-6736(16)00625-5

33. Macnamara FN (1954) Zika virus: a report on three cases of human infection during an epidemic of jaundice in Nigeria. Trans R Soc Trop Med Hyg 48(2):139-45

34. Martines RB, Bhatnagar J, Keating MK, et al (2016) Notes from the Field: Evidence of Zika Virus Infection in Brain and Placental Tissues from Two Congenitally Infected Newborns and Two Fetal Losses - Brazil, 2015. MMWR Morb Mortal Wkly Rep 65 (6):159-60

35. Mécharles S, Herrmann C, Poullain P, et al (2016) Acute myelitis due to Zika virus infection. Lancet S0140-6736(16)00644-9

36. Mlakar J, Korva M, Tul N, et al (2016) Zika Virus Associated with Microcephaly. N Engl J Med 374(10):951-8

37. Musso D (2015) Zika Virus Transmission from French Polynesia to Brazil. Emerg Infect Dis 21(10):1887

38. Musso D, Nhan T, Robin E, et al (2014) Potential for Zika virus transmission through blood transfusion demonstrated during an outbreak in French Polynesia, November 2013 to February 2014. Euro Surveill. 2014 19(14)

39. Musso D, Roche C, Robin E, et al (2015) Potential sexual transmission of Zika virus. Emerg Infect Dis 21(2):359-61

40. Oehler E, Watrin L, Larre P, et al (2014) Zika virus infection complicated by Guillain-Barre syndrome--case report, French Polynesia, December 2013. Euro Surveill 19(9)
41. Organisation Mondiale de la Santé (OMS) (2016) Déclaration sur la 1ère réunion du Comité d'urgence du Règlement sanitaire international concernant le virus Zika 2016 - $1^{\mathrm{er}}$ février 2016

42. Petersen EE, Staples JE, Meaney-Delman D, et al (2016) Interim Guidelines for Pregnant Women During a Zika Virus Outbreak United States, 2016. MMWR Morb Mortal Wkly Rep 65(2):30-3

43. Production Team REDUAS, Coordinator Dr. Medardo Avila Vazquez (2016) Report from Physicians in the Crop-Sprayed Villages regarding Dengue-Zika, microcephaly and mass-spraying with chemical poisons - February 3, 2016, 6 pp

44. Roa M (2016) Zika virus outbreak: reproductive health and rights in Latin America. Lancet 387(10021):843

45. Salvador FS, Fujita DM (2015) Entry routes for Zika virus in Brazil after 2014 world cup: New possibilities. Travel Med Infect Dis 14(1):49-51

46. Sarno M, Sacramento GA, Khouri R, et al (2016) Zika Virus Infection and Stillbirths: A Case of Hydrops Fetalis, Hydranencephaly and Fetal Demise. PLoS Negl Trop Dis 10(2):e0004517

47. Schuler-Faccini L, Ribeiro EM, Feitosa IM, et al (2016) Possible Association Between Zika Virus Infection and Microcephaly Brazil, 2015. MMWR Morb Mortal Wkly Rep 65(3):59-62

48. Secretarias de Saúde dos Estados e Distrito Federal do Brasil (2016) Saúde investiga 4.222 casos suspeitos de microcefalia no país. Portal da Saúde [Internet]

49. Tang H, Hammack C, Ogden SC, et al (2016) Zika virus infects human cortical neural progenitors and attenuates their growth. Cell Stem Cell 18:1-4

50. Tetro JA (2016) Zika and microcephaly: Causation, correlation, or coincidence? Microbes Infect S1286-4579(16)00008-3

51. Venturi G, Zammarchi L, Fortuna C, et al (2016) An autochthonous case of Zika due to possible sexual transmission, Florence, Italy, 2014. Euro Surveill 21(8)

52. Victora CG, Schuler-Faccini L, Matijasevich A, et al (2016) Microcephaly in Brazil: how to interpret reported numbers? Lancet 387(10019):621-4

53. World Health Organization (WHO) (2016) Breastfeeding in the context of Zika virus - Interim guidance. 25 February 2016. 2 p

54. World Health Organization (WHO) (2016) Psychosocial support for pregnant women and for families with microcephaly and other neurological complications in the context of Zika virus- Interim guidance for health-care providers, $18 \mathrm{pp}$

55. World Health Organization (WHO) (2016) Risk Communication and Community Engagement for Zika Virus Prevention and Control - A Guidance and Resource Package for Country Offices for Coordination, Planning, Key Messages and Actions. March 2016, $22 \mathrm{p}$

56. World Health Organization (WHO) (2016) Zika virus, microcephaly and Guillain-Barré syndrome. Situation report. 4 March 2016, 15 pp 